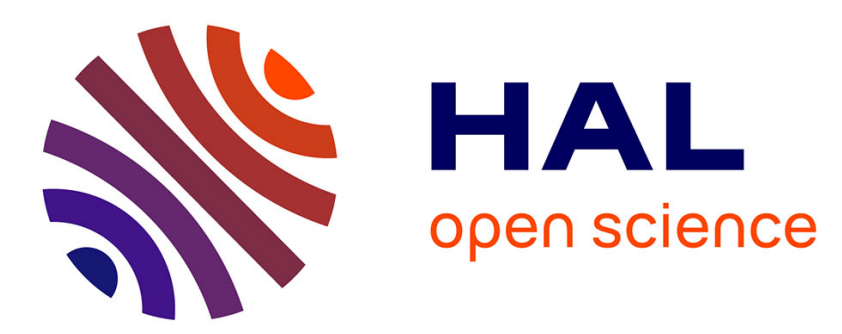

\title{
Inventory policies analysis under demand patterns and lead times constraints in a real supply chain
}

Giuseppe de Sensi, Francesco Longo, Giovanni Mirabelli

\section{To cite this version:}

Giuseppe de Sensi, Francesco Longo, Giovanni Mirabelli. Inventory policies analysis under demand patterns and lead times constraints in a real supply chain. International Journal of Production Research, 2008, 46 (24), pp.6997-7016. 10.1080/00207540701528776 . hal-00512995

\section{HAL Id: hal-00512995 \\ https://hal.science/hal-00512995}

Submitted on 1 Sep 2010

HAL is a multi-disciplinary open access archive for the deposit and dissemination of scientific research documents, whether they are published or not. The documents may come from teaching and research institutions in France or abroad, or from public or private research centers.
L'archive ouverte pluridisciplinaire HAL, est destinée au dépôt et à la diffusion de documents scientifiques de niveau recherche, publiés ou non, émanant des établissements d'enseignement et de recherche français ou étrangers, des laboratoires publics ou privés. 


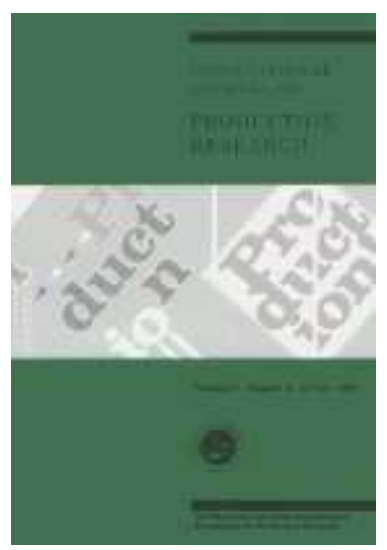

\section{Inventory policies analysis under demand patterns and lead times constraints in a real supply chain}

\begin{tabular}{|c|c|}
\hline Journal: & International Journal of Production Research \\
\hline Manuscript ID: & TPRS-2007-IJPR-0071.R1 \\
\hline Manuscript Type: & Original Manuscript \\
\hline $\begin{array}{r}\text { Date Submitted by the } \\
\text { Author: }\end{array}$ & 11-Jun-2007 \\
\hline Complete List of Authors: & $\begin{array}{l}\text { De Sensi, Giuseppe; University of Calabria, Mechanical Department } \\
\text { Longo, Francesco; University of Calabria, Mechanical Department } \\
\text { Mirabelli, Giovanni; University of Calabria, Mechanical Department }\end{array}$ \\
\hline Keywords: & $\begin{array}{l}\text { INVENTORY CONTROL, SUPPLY CHAIN MANAGEMENT, SIMULATION } \\
\text { APPLICATIONS, DESIGN OF EXPERIMENTS }\end{array}$ \\
\hline Keywords (user): & Multi-echelons supply chain, Stochastic uncertainty \\
\hline
\end{tabular}

\section{scholarONE" \\ Manuscript Central}




\title{
Inventory policies analysis under demand patterns and lead times constraints in a real supply chain
}

\author{
G. DE SENSI, F. LONGO*, G. MIRABELLI \\ Mechanical Department, University of Calabria \\ 87036 Rende (CS), Italy
}

\begin{abstract}
This paper reports a study on a real three-echelon supply chain operating in the beverage sector. The authors, starting from the actual supply chain configuration, propose a detailed study of the inventory systems. The test of a comprehensive set of different operative scenarios, in terms of customers' demand intensity, customers' demand variability and lead times, becomes a powerful tool for inventory systems analysis along the supply chain. The main objective is the comparison of the actual supply chain configuration with optimistic and pessimistic scenarios for studying the behaviour of different inventory control policies and optimizing the inventory system of each supply chain node. Due to the dynamically changing and stochastic behaviour of the supply chain variables as well as the complex interactions among its actors, the authors have been pushed to implement an advanced simulation model supported by a 'well planned' experimental design. In addition the paper aims to underline the research effort for conceptualizing, modelling, validating and simulating a real stochastic supply chain.
\end{abstract}

Keywords: Inventory Control Policies; Multi-echelon; Supply chain; Stochastic Uncertainty; Simulation

\section{Introduction}

The business globalization has transformed the modern companies from independent entities to extended enterprises that strongly cooperate with all supply chain actors. Each supply chain manager aims to reach the key objective of an efficient supply chain: 'the right quantity at the right time and in the right place'.

To this end, each supply chain node (suppliers, manufacturers, warehouses, retailers, etc.), by using different methodologies for planning and scheduling, carries out various processes and activities for guarantying goods and services to the final customers (refer to Kreipl and Pinedo, 2004, for a detailed overview on theory and practice of planning and scheduling in supply chains). The competitiveness of each supply chain member depends by its capability to activate and manage change processes, in correspondence of optimistic and pessimistic scenarios, to quickly capitalize the chances given by market. Such capability is a critical issue for improving the performance of the 'extended enterprise' and it must take into account the complex interactions among the various nodes of the supply chain. The evaluation of correct trades-off between conflicting factors, such as inventory reduction (control policies) and fill rates, customers' satisfaction (service levels) and transportation cost (lead times), sales loss and inventory costs, is the complex task of an efficient supply chain manager.

\footnotetext{
* Corresponding Author. Email: f.longo@unical.it
} 
As expressed in De Sensi et al. (2006), due to the close connection among the different components of the logistics network as well as the stochastic behaviour of supply chain variables, the analytical approach is not always the most suitable tool for modelling and analyzing real supply chain scenarios. In this research work we propose a simulation based tool for facing the complexity of the inventory systems of a three echelon real supply chain.

The following literature overview reports several research works, both theoretical and applied, on supply chain inventory systems. Many research works identify, as critical parameters in defining the optimal inventory control policies, the customers' demand pattern, the lead times and the information sharing. Most of the cases propose a comparative analysis of different operative scenarios or configurations (Modelling \& Simulation is often used as what if analysis or cognitive tool).

Specific studies on the base stock policy are reported in Roundy and Muckstadt (2000), Graves (1999) and Parker and Kapuscinski (2004). In the first case the authors use the base stock policy evaluating policy parameters and obtaining a good approximation to the optimal policy. In the second case the base stock policy is applied in correspondence of different operative scenarios (demand pattern variation). In the third case the authors demonstrate that the base stock policy, in a two echelon supply chain, obtains the best performance (respect to the other inventory policies) if downstream stages capacity is lower than upstream ones.

Similar studies have been carried out for other inventory control policies. The influence on supply chain performance of the most applied inventory policies (economic order quantity with stationary demand and dynamic economic lot-size with non stationary demand) is reported in Zipkin (2000). Bertsimas and Thiele (2006) propose an approach that takes into consideration demand uncertainty and provides as results insights about the optimal policy (considering an optimal trade off between performance and protection against uncertainty). Other works related to inventory systems are reported in Simchi-Levi et al. (2000), Stadtler and Kilger (2000) and Chopra and Meindl (2001).

For giving to supply chain managers the right managerial insights the research efforts must be focalized along the entire supply chain as more as possible (as mentioned earlier the supply chain manager has to take into account the concept of extended enterprise). In addition to the literature before cited, many authors provided an enlarged framework for inventory systems analysis.

Wikner et al. (1991) implements five different supply chain strategies:

- tuning of order policy parameters;

- reduction of the delivery delays in each stage of the supply chain;

- distribution echelon elimination;

- better decisions rules;

- improving of the information flow separating customers' real orders from the orders emitted for the safety stock.

The authors propose a model composed by a single production plant, various distribution centres and retailers, each one with an own inventory policy. They use simulation for evaluating the best inventory policies that minimize demand fluctuation along supply chain. T. S. Chan and H. K. Chan (2005) propose a similar approach. They test different supply chain configurations, by means of Modelling \& Simulation, to determine the best one.

Such enlarged supply chain scenario often includes the information sharing upstream and downstream the supply chain as key element for inventory system optimization. Li et al. (2001) focalize on different supply chain scenarios, obtained by changing purchase orders, customers' demand, inventory policy and information flow. The authors find out that, under low demand intensity, the information sharing allows lower inventory costs as well as higher fill rate. Kelle and Milne (1999) demonstrate that in a supply chain, the (s, S) policy, jointly with smaller quantity for each order, higher number of purchase orders and cooperation among supply chain stage (information sharing), reduces demand variability along the supply chain. The evaluation, by means of simulation, of the impact of inventory system variations and information sharing on demand 
variability along the supply chain, is also the focus of Towill (1991). All the research studies involving supply chain information sharing allow to gain knowledge and confidence about supply chain but they analyze unrealistic scenarios (usually there is no information sharing among supply chain node due to competitiveness issues).

The study of inventory systems in real stochastic supply chains is one of the major concerns in today supply chain management. As soon as the number of parameters affecting supply chain performance becomes high and the objective becomes the whole supply chain analysis, simulation plays a more critical role in finding the optimal trade off among the involved variables (inventory policies, transportation cost, lead times, demand patterns, customers' satisfaction) as reported in Chang and Makatsoris (2001). To this end Modelling \& Simulation based approach is jointly used with statistics techniques such as Design of Experiment and Analysis of Variance, (Longo et al. 2005, Suwanruji and Enns 2006) or in combination with artificial intelligence techniques (Giannoccaro and Pontrandolfo, 2002)

The state of art overview highlights that (i) Modelling and Simulation in combination with statistic techniques is usually used for analyzing supply chain scenarios (different combinations of critical parameters), (ii) there is a lack in the research studies on inventory systems of real multiechelon stochastic supply chain, considering a complete set of operative scenarios regarding customers' demand intensity, customers' demand variability, lead times and the impact of such scenarios on multiple performance measures. In such a context, research works based on analytical approaches are characterized by simplifying assumptions, studies based on Modelling \& Simulation consider a limited number of operative scenarios, or a limited number of inventory policies, or they are based on theoretical case study or, at last, they consider only one performance measure.

The contribution of this paper is a focus on a real three-echelon stochastic supply chain operating in the beverage sector. Note that Modelling \& Simulation has been used for reaching the main objective of the paper that is the optimization of the inventory system of each supply chain node analyzing a complete set of inventory policies as well as a comprehensive set of operative scenarios.

In addition the paper aims to underline the research effort in following scientific methods for conceptualizing, modelling, validating and simulating a real stochastic supply chain.

\section{Steps of the study}

We have adopted, for our study, the same set of steps of a simulation study described by Banks (1998). The list is as follows:

- problem formulation;

- $\quad$ setting of objectives;

- model conceptualization;

- data collection;

- model translation;

- verification, simulation run length and validation;

- experimental design;

- production runs and analysis.

We have already introduced the problem formulation and the objectives of the research study highlighting, by means of the state of art overview, the paper contribution to the literature.

Before getting into the details of the study let us give a brief summary of the paper. The following sections retrace the remaining steps of the study. Section 3 is about model conceptualization and data collection, section 4 describes the fundamental steps followed during the modelling phase (model translation) and the model verification and validation. Section 5 deals with the experimental design, simulation results and output analysis. The last section reports conclusions as well as research guidelines for future works. 


\section{Model conceptualization}

As mentioned earlier, the authors analyze a three-echelon supply chain operating in the beverage sector. The supply chain consist of five manufacturing plants, two distribution centres (here in after DCs), ten big retailers and more than one hundred items (see figure 1).

\section{<figure 1 should be inserted approximately here $>$}

Each manufacturing plant produces a certain mix of products activating different production processes. Plants are 'make to order' systems, they don't have warehouses, thus, items are sent to DCs just after the production. The transportation activities are carried out by a third party logistics (3PL) according to low variable lead times. In our conceptual model we assume constant transportation lead times. We use an estimate of the lead time standard deviation only for safety stock calculations.

The DCs use a continuous review policy, $(\mathrm{r}, \mathrm{R})$, with fixed review period. Consider the distribution centre $i$ and the item $j$, the (r,R) policy places an order every time the inventory position falls below the reorder point $r_{i, j}(t)$. The ordered quantity will bring the inventory position to the target level $R_{i, j}(t)$. The inventory position, $I P_{i, j}(t)$, is the on hand inventory, plus the quantity already on order, minus the quantity to be shipped. The reorder point $r_{i, j}(t)$ is the lead time demand (evaluated as the daily demand, $D_{i, j}(t)$, averaged over the last $T$ periods, times the lead time, $L T_{i, j}(t)$ ) plus the safety stock, $S S_{i, j}(t)$, as expressed in equation (1).

$$
r_{i, j}(t)=L T_{i, j}(t) \frac{\sum_{t}^{t+T-1} D_{i, j}(t)}{T}+S S_{i, j}(t)
$$

Let $\mathrm{T}_{\mathrm{p}}$ be the review period, the target level $R_{i, j}(t)$ is the sum of the average demand over the review period and reorder point $r_{i, j}(t)$ :

$R_{i, j}(t)=\frac{\sum_{t}^{t+T p-1} D_{i, j}(t)}{T_{p}}+r_{i, j}(t)$

The order emission condition and the quantity to be ordered, $Q i, j(t)$, respectively follow equations (3) and (4).

$I P_{i, j}(t)<r_{i, j}(t)$

$Q_{i, j}(t)=R_{i, j}(t)-I P_{i, j}(t)$

Even though the two distribution centres use the same inventory control policy, they do not use the same demand forecasting methodology. The first distribution centre uses the moving average methodology; the second one uses the single exponential smoothing. Every day the DCs try to satisfy retailers demand. Unsatisfied demand is recorded for performance indexes calculation. Items distribution is made giving to each retailer the same priority. In case of stock out the available quantity is proportionally subdivided among the retailers. Transportations between DCs and retailers are assured by a 3PL, guarantying low variable lead times. As in the case of plants we assume, in our conceptual model, constant lead times and we use an estimate of the lead time standard deviation for safety stock calculations. 
$Q_{i, j}(t)=L T D_{i, j}(t)+S S_{i, j}(t)-I P_{i, j}(t)$

Customers' demand is a stochastic process, thus, real data has been collected and analyzed. Please refer to the next section for further information about data input analysis. Each retailer calculates the demand forecasting according to moving average methodology.

Note that we propose a simulation study, thus, in addition to the actual inventory control policies, new inventory control policies can be tested and analyzed. The retailers' policy adopts a constant safety stock; integration to this policy is a safety stock that takes into consideration both the demand standard deviation and the lead time standard deviation. We can write:

$$
S S_{i, j}(t)=s_{i, j}(t) *\left\lfloor L T_{i, j}(t)+s l_{i} * s_{i}\right\rfloor
$$

- $\quad s_{i, j}(t)$, estimate of the daily demand standard deviation at the supply chain node $i$, for product $j$;

- $s_{i}$, estimate of the lead time standard deviation at the supply chain node $i$;

- $s l_{i}$, factor expressing the service level that should be provided at the supply chain node $i$ with the safety stock.

As integration to the policy used by DCs we propose an optimized review period based approach. The optimized review period tries to minimize, on the basis of demand forecast, the inventory cost of each item, $I C_{i, j}(t)$.

$$
I C_{i, j}(t)=\frac{C_{P O E, i j}+C_{S T, i j} * \sum_{t}^{t+T^{*}-1}(t-1) * D F_{i, j}(t)}{\sum_{t}^{t+T^{*}-1} D F_{i, j}(t)}=M I N
$$

- $C_{P O E, i j}$, total cost for purchase order emission (order emission, transportation and reception) at supply chain node $i$ for item $j$

- $C_{S T, i j}$, total cost for storage (per item and per unit of time) at the supply chain node $i$ and for item $j$;

- $\quad D F_{i, j}(t)$, demand forecast at the supply chain node $i$ and for item $j$;

- $T^{*}$, optimized review period.

Equation (8) is iteratively calculated until the minimum condition is reached. The reorder point, the target level, the order emission condition and the quantity to be ordered, respectively follow equations (1) - (4), with $T_{p}=T^{*}$.

In order to test a comprehensive set of inventory policies, the simulation model will be implemented considering for each supply chain node (both retailers and DCs) its own policy plus the three remaining policies. Here in after let us identify the actual retailers' policy with ICP1, the integration of such policy with dynamic safety stock, ICP2, the actual DCs' policy with ICP3 and the policy based on optimal review period with ICP4. 


\subsection{Data collection}

Data collection in a whole supply chain is one of the most critical issues. The random behaviour of some variables makes the supply chain a stochastic system. As reported by Banks (1998), for each element in a system being modelled, the simulation analyst must decide on a way to represent the associated variables. The Data Collection step takes care of collecting data in each node of the supply chain as well as finds the most suitable computer representation for such data.

Usually there are three different choices: (i) data are deterministic or data are considered as deterministic, (ii) a distribution probability is fitted to empirical data and (iii) the empirical distribution of the data is directly used in the simulation model.

The supply chain is characterized both by deterministic data and by stochastic data (we mean both numerical data and inputs that drive the logic of the supply chain). The second and the third choices have been adopted for representing, in the simulation model, supply chain stochastic variables.

In case of stochastic variables and distributions fitting, the procedure for input data analysis is the classical procedure proposed by many statistics references as well as implemented in numerous commercial software: (i) starting from a histogram of the data, one or more candidate distributions are hypothesized, (ii) for each distribution the characterizing parameters are estimated, (iii) a goodness of fit test is preformed, (iv) at last, the best distribution is chosen. For any additional information on input data analysis for simulation studies please refer to Johnson et al. (1992, 1994, 1995) and D'Agostino and Stephens (1986).

Table 1 consists of a list of the most important variables and information collected for each plant, distribution centre and retailer. Most of the data have been obtained using companies' informative systems. The data in italicized style are characterized by stochastic behaviour.

As example of the procedure followed for input data collection and analysis, consider the market demand arrival process. Customers' inter-arrival times have been collected and fitted using the above mentioned procedure for each retailer.

$<$ table 1 should be inserted approximately here>

Let us focus on the retailer \#1. Starting from the histogram of the data (based on 21 classes, see figure 2) four different distributions have been hypothesized: Erlang, Weibull, Negative Exponential and Lognormal. The collected data allow the calculation of the distributions parameters, summarized in table 2. The successive step is the goodness of fit test. Note that we deal with a large sample so the Chi-Square test performs better than Ardenson-Darling and KolmogorovSminorv tests. As well known from statistics theory if the Chi Statistics is lower than the Chi Value, the distribution accurately fit the real data. The Result column in table 2 shows that the Erlang and Negative Exponential distributions perform a good fit of the data. In presence of two or more available distributions, the choice falls on the distributions with lowest Chi Statistics. In our case the Negative exponential distribution has been selected for representing the customers' inter-arrival times for retailer \#1.

$<$ table 2 should be inserted approximately here $>$

$<$ figure 2 should be inserted approximately here>

As final result we obtained, for each retailer, a Poisson process (numerous scientific works confirm such results for inter-arrival times). Due to high number of items, the data regarding the quantity required by customers have been analyzed in terms of minimum, average and maximum values (triangular distributions). Each customer can require each type of good; items mixture is represented in the simulation model with empirical distributions. Lead times have been fitted with normal distributions. Plants process times and setup times use the empirical distributions. Table 3 
consists of statistic distributions and parameters related to collected data. Note that the triangular distribution is reported only for item \#1. Analogous information are available for each item.

$<$ table 3 should be inserted approximately here $>$

\section{Conceptual model translation}

The supply chain conceptual model and the collected data have been translated in a simulation model using eM-Plant, a simulation model developed by Tecnomatix Technologies. eM-Plant proposes a typical object oriented modelling approach. Library objects, such as resources and entities, are used for modelling any real system or scenario. Our experience with eM-Plant simulation models (Longo et al. 2006), has highlighted two different problems (in modelling supply chain with the traditional modelling approach). As soon as the number of entities, flowing in the simulation model, becomes too high the time required for executing a simulation run becomes unacceptable (simulation models computationally too heavy). In addition, sometimes library objects do not permit a 'realistic' representation of the system being modelled.

To avoid such problems we propose an advanced modelling approach based on programming code, tables and event generators. eM-Plant provides the user with a programming language (Simple++). Such programming language is used, by means of methods, for modelling the supply chain. In our approach the flow of entities is substituted by information recorded in tables. The events, usually generated by entities flow, are replaced by events generated with Event Generators objects (provided by eM-Plant libraries). The outcome is a time efficient (shorter time for executing a simulation run) and flexible (each class of the simulation model can be accessed and modified by code) simulation model.

However, the modelling approach as well as the architecture of the simulation model is not the objectives of the paper. Even tough the simulation model, thanks to its architecture, is capable of modelling a generic or theoretical supply chain, in this case it is applied to a real supply chain for studying the inventory systems under different operative scenarios.

The main frame of the simulation model recreates the logical connections among the various nodes of the supply chain (see figure 3). The model provides a graphic user interface for inserting input parameters (process times, setup times, lead times, inter-arrival time, quantity etc.).

\section{$<$ figure 3 should be inserted approximately here $>$}

In order to reach the objective of the paper we chose two different performance measures: the fill rate and the on hand inventory. The fill rate is calculated for each store and distribution centre (at the end of the day) as the ratio between the number of satisfied orders and the total number of received orders. Note that in case of discrepancy between quantity required by customers and quantity provided by retailers, the order is considered as unsatisfied. Thus, lower values of fill rate don't necessarily mean low retailer's efficiency. For sure the higher is the fill rate the higher is the service level provided to customers, but we are not taking into consideration the quantity (difference between quantity required and quantity effectively provided), that makes an order unsatisfied.

The on hand inventory is calculated (at the end of the day) as mean value between the on hand inventory before the business hour and the on hand inventory after the business hour.

\subsection{Verification, simulation run length and validation of the simulation model}

The accuracy and the quality throughout a simulation study are assessed by conducting verification and validation processes (Balci 1998). The American Department of Defence Directive 5000.59 defines verification and validation as follows. Verification is the process of determining that a 
model implementation accurately represents the developer's conceptual description and specifications. Obviously this step is strictly related to model translation. Validation is the process of determining the degree to which a model is an accurate representation of the real world from the perspective of the intended use of the model. Problems during the validation phase can be attributed to model conceptualization or data collection. In our case, according to the published literature, the verification and validation has been conducted throughout the entire lifecycle of the simulation study and using both dynamic and informal verification and validation techniques.

4.1.1 Verification. The simulator verification has been made using a dynamic technique (debugging). As explained in Dunn (1987), Debugging is an iterative process that aims to find model errors and to improve the model correcting the errors. The model is tested for revealing the presence of bugs. The causes of each bug must be correctly identified. The model is opportunely modified and tested (once again) for ensuring errors elimination as well as for detecting new errors. All the methods (routines written in Simple++) have been iteratively debugged line by line, detecting and correcting all the errors. Errors detected during the simulation study life cycle were due to: misunderstanding or numerical error on input data, tables and spreadsheet indexes management, events list organization and management. In addition, before model translation, all the logics governing supply chain have been discussed with the companies' experts.

4.1.2 Simulation run length. Before getting into details of simulation model validation, we need to speak about the simulation run length. The length of a simulation run is an information used for validation, for design of experiments and simulation results analysis. Such length is the correct trade-off between results accuracy and time required for executing the production runs. The run length has been correctly determined using the mean square pure error analysis $\left(\mathrm{MS}_{\mathrm{PE}}\right)$.

As well known the mean square of the experimental error must have a knee curve trend. As soon as the simulation time goes by, the standard deviation of the experimental error (due to statistic and empirical distributions implemented in the simulation model) becomes smaller. The final value has to be small enough to guarantee high statistical result accuracy. Notice that we are speaking about the experimental error of the supply chain performance indexes (fill rate and average on hand inventory).

Consider that the performance measures are calculated for each supply chain node, thus, the $\mathrm{MS}_{\mathrm{PE}}$ analysis has to be repeated for each supply chain node and for each performance measure. The $\mathrm{MS}_{\mathrm{PE}}$ curve that takes the greatest simulation time for obtaining negligible values of the mean squares defines the simulation run length. Figure 4 shows the fill rate $\mathrm{MS}_{\mathrm{PE}}$ curve of distribution centre \#2 that takes the greatest simulation time. After 400 days the $\mathrm{MS}_{\mathrm{PE}}$ values are negligible and further prolongations of the simulation time do not give significant experimental error reductions.

$<$ figure 4 should be inserted approximately here $>$

4.1.3 Validation. Choosing for each simulation run the length evaluated by means of $\mathrm{MS}_{\mathrm{PE}}$ analysis (400 days) the validation phase has been conducted using the Face Validation (informal technique). For each retailer and for each distribution centre the simulation results, in terms of fill rate, have been compared with real results. For a better understanding of the validation procedure let us consider the retailer \#1. Figure 5 shows six different curves, each one reporting the retailer \#1 fill rate versus time (days). In the graphs there is one real curve and five simulated curves. Note that during the validation process the simulation model works under identical input conditions of the real supply chain.

$<$ figure 5 should be inserted approximately here $>$ 
The plot has been shown to the company's experts asking to make the difference between the real curve and the simulated curves on the basis of their estimates (obviously showing all the curves with the same colour). The experts weren't unable to make such difference, testifying the validation of the simulation model. The Face Validation technique has been applied for the remaining retailers as well as for each distribution centre. Further results in terms of fill rate confidence intervals have been analyzed. We concluded that, in its domain of application, the simulation model recreates with satisfactory accuracy the real system.

\section{Experimental design}

In addition to the actual supply chain configuration, the simulation model adds new features in terms of inventory control policies, market demand pattern and lead time. Starting from the inventory control policies used by retailers and DCs we propose a time dependent safety stock as well as the optimization of the review period on the basis of the inventory cost of each item. Maintaining the initial policies and implementing the new policies as well, the simulation model provides each supply chain node with four control policies based on continuous review.

Keeping fixed the customers' inter-arrival distribution, the demand intensity and variability have been modified to create alternative scenarios. The actual demand intensity and variability are considered as medium level, low demand intensity and variability as well as high demand intensity and variability are considered.

Additional scenarios also regard the lead time from DCs to lead time. The actual lead time is considered as medium level, an optimistic (lower lead time) and a pessimistic (higher lead time) scenario are considered.

The investigation and comparison of all possible scenarios requires a correct design of experiments. Note that there are four different factors: (1) inventory control policy; (2) lead time; (3) demand intensity; (4) demand variability. Each factor has different levels: four different inventory control policies, three lead time values, three demand intensity levels, three demand variability levels. Table 4 consists of a summary of factors and levels.

$<$ table 4 should be inserted approximately here $>$

Factors levels combinations creates a comprehensive set of different operative scenarios, in terms of customers' demand intensity, customers' demand variability and lead times. All factors levels combinations give as result 108 different scenarios for analyzing and optimizing (best supply chain configurations) the inventory systems along the supply chain.

Each scenario has been simulated for 400 days (according to the $\mathrm{MS}_{\mathrm{PE}}$ analysis) and replicated 4 times using different seed numbers for random numbers generation (4 replications are required for guarantying reliable statistic results).

\subsection{Production runs and analysis}

Simulation results, for each factors levels combinations, are expressed in terms of average fill rate and average on hand inventory (calculated as mean value of the average on hand inventory of all the items). Simulation results are available for each retailer as well as for each distribution centre.

The huge quantity of simulation results allows the analysis of a comprehensive set of operative scenarios. Let us consider the simulation results regarding the retailer \#1, we have extrapolated the following scenarios according to the company experts' requests.

1. pessimistic, actual and optimistic scenarios in terms of demand intensity with actual lead time and average demand variability (table 5 and figure 6);

2. pessimistic, actual and optimistic scenarios in terms of demand variability with actual lead time and actual demand intensity (table 6 and figure 7); 
3. pessimistic, actual and optimistic scenarios in terms of lead time with actual demand intensity and actual demand variability (table 7 and figure 8).

The inventory control policies implemented in the simulation model shows different behaviours in correspondence of each scenario.

Table 5 consists of fill rate and average on hand inventory (enclosed in parenthesis) results of the first scenario. Keeping fixed the demand intensity the highest fill rate is given by the inventory control policy ICP4. The ICP2 is characterized by the worst fill rate in all the cases. The lowest value for the average on hand inventory is obtained with the policy ICP4.

Keeping fixed the inventory control policy, as soon as the demand intensity increases there is a strong reduction of the fill rate. Notice that in correspondence of high intensity the performance of the inventory control policy based on the review period optimization, ICP4, is comparable with the classic policy based on fixed review period (ICP3). The review period optimization is based on forecast demand and the error in demand forecast increases with of high quantities. The higher is the demand intensity the higher is the average on hand inventory. Once again the best inventory control policy is the ICP4 even if the policy ICP3 gives similar results. Note that ICP3 is currently used only by DCs it could be easily implemented by each retailer for increasing fill rate and decreasing on hand inventory.

$<$ table 5 should be inserted approximately here>

$<$ figure 6 should be inserted approximately here $>$

Table 6 consists of fill rate and average on hand inventory results of the second scenario. On the contrary of the previous scenario the optimistic case is characterized by low demand variability. The ICP2 gives the worst performance. Note the similar behaviour of ICP3 and ICP4. The policy based on the optimized review period shows a better behaviour in correspondence of low demand variability. In fact, the demand forecast usually gives greater error with high demand variability (remember that the optimization period is based on demand forecast). As in the previous case the retailers should change the inventory control policy ICP1 with the ICP3 or ICP4. The higher is the demand variability the higher is the average on hand inventory. The policies ICP3 and ICP4 give the best performance in terms of average on hand inventory. The best policy with low demand variability is the ICP4, with the actual demand variability ICP3 and ICP4 show the same performance, finally with high demand variability ICP3 shows the best behaviour.

$<$ table 6 should be inserted approximately here $>$

$<$ figure 7 should be inserted approximately here $>$

Table 7 consists of fill rate and average on hand inventory results of the third scenario. Such scenario investigates the effect of different lead times on fill rate and average on hand inventory. The positive performance of the policies ICP4 and ICP3 is still confirmed. The major issue is the fill rate reduction passing from 1 day lead time to 3 and 5 days lead time. Note that with ICP1 and ICP2 the fill rate reduction is respectively $4.4 \%$ and $17.2 \%$ (from 1 to 3 days lead time), $8.1 \%$ and $25.7 \%$ (from 3 to 5 days lead time). The reduction with ICP3 and ICP4 is remarkable smaller, $2.1 \%$ and $1.5 \%$ (from 1 to 3 days lead time), $1.2 \%$ and $2.4 \%$ (from 3 to 5 days lead time). Thus, ICP3 and ICP4 show a better behaviour than ICP1 and ICP2 both in absolute and in relative terms. The results in terms of average on hand inventory show that smaller quantity for each order and higher number of purchase orders decreases the inventory levels.

$<$ table 7 should be inserted approximately here>

$<$ figure 8 should be inserted approximately here> 
The results above presented regard the retailer \#1. The analysis of each scenario allows to evaluate the behaviour of the inventory control policies as well as to find out the best performance of the inventory systems in terms of fill rate and average on hand inventory. The new scenarios are compared with the actual scenario in terms of what-if analysis. Similar results have been obtained for each supply chain node, both retailers and distribution centres, analyzing and optimizing the inventory systems along the supply chain.

\section{Conclusions and future research}

A real three echelon supply chain operating in the beverage sector has been considered. Due to its stochastic behaviour, the supply chain has been studied using an advanced Modelling \& Simulation based approach supported by a well planned design of experiments. The initial phases of the study have required a strong cooperation with supply chain managers for correctly defining the conceptual model, for input data collection, for model translation, verification and validation.

Having obtained a simulation model that recreates with satisfactory accuracy the real system, we conducted a comprehensive investigation of the inventory systems along the supply chain under different demand patterns and lead times constraints. The actual demand intensity, demand variability and lead times are considered as medium levels and integrated with lower and higher levels. In addition three inventory control policies have been added to each retailer and each distribution centre. For studying inventory systems efficiency two different performance measures have been implemented: fill rate and average on hand inventory.

The comparison of the actual supply chain configuration with pessimistic and optimistic scenarios, in terms of critical factors (customers' demand intensity, customers' demand variability and lead times) is a powerful tool for analyzing the behaviour of different inventory control policies. The analysis of each scenario for each supply chain node allows to evaluate the impact of each factor on fill rate and on average on hand inventory, addressing the choice of the best inventory control policy as well as the best supply chain configuration.

Due to the high flexibility of the simulation model (and considering that the model is capable of recreating with high accuracy the real supply chain) further researches are still on going for analyzing another critical issue: the transportation between the different supply chain nodes. The simulation model has been updated by adding an additional echelon to the supply chain (final customers, more than one hundred stores). The attention will be focalized on transportation from the distribution centres to big retailers and from big retailers to stores. The simulation approach will be integrated with artificial intelligence techniques (genetic algorithms and ants theory) for finding out the best supply chain configurations in terms of transportation modalities and routes. Note that transportation modalities are strictly connected to inventory systems management. In effect, transportation modalities perform differently in relation to inventory policies and to specific system parameters (such as maximum waiting time before trucks departures, security factors on demand forecasts for transportation planning and so on). Such work has to be regarded as an additional effort in considering research studies on real multi-echelon supply chain.

\section{References}

Balci, O., Verification, Validation and Testing, in Handbook of Simulation, edited by J. Banks, pp. 335-393, 1998 (Wiley Interscience: New York).

Banks, J., Handbook of simulation, 1998 (Wiley Interscience: New York).

Bertsimas, D., A. Thiele, A robust Optimization approach to Inventory Theory, Oper. Res., 2006, $\mathbf{5 4}(1), 150-168$.

Chan, T. S., H. K. Chan, Simulation modelling for comparative evaluation of supply chain management strategies, Int. J. Adv. Manuf. Technol, 2005, 25, 998 - 1006. 
Chang, Y., H. Makatosoris, Supply chain modelling using simulation, Int. J. Simulation, 2001, 2(1), $24-30$.

Chopra, S., P. Meindl, Supply chain management - Strategy, planning, and Operation, 2001, (Prentice-Hall: New Jersey).

D’Agostino R.B., M.A. Stephens, Goodness of fit techniques, 1986, (Marcel Dekker: New York).

Daganzo, C. F., On the stability of the supply chains, Op. Res., 2004, 52(6), $909-921$.

De Sensi, G., F. Longo, G. Mirabelli, E. Papoff, Ants colony system for supply chain routes optimization, in the Harbour, Maritime \& Multimodal Logistics Modelling and Simulation Conference, 2006, pp. $763-768$.

Department of Defense, Deputy under Secretary of Defence, DoD modelling and simulation (M\&S) management, DoD Directive 5000.59, 1994

Dunn R.H., The quest for software reliability, in Handbook of software quality assurance, edited by G.G. Schulmeyer and J.I. McManus, pp. 342 - 384, 1987 (Van Nostrand Reynold: New york).

Giannoccaro, I., P. Pontrandolfo, Inventory management in supply chains: a reinforcement learning approach, Int. J. Prod. Econ., 2002, 78, 153 - 161.

Graves, S. C., A single inventory model for a non-stationary demand process, Manuf. Serv. Op. Manag., 1999, 1, 50 - 61.

Johonson N.L., S. Kotz, A.W. Kemp, Univariate Discrete Distributions, $2^{\text {nd }}$ ed., 1992, (Houghton Mifflin: Boston).

Johonson N.L., S. Kotz, N. Balakrishnan, Continuous Univariate Distributions, Vol. 1, $2^{\text {nd }}$ ed., 1994, (Houghton Mifflin: Boston).

Johonson N.L., S. Kotz, N. Balakrishnan, Continuous Univariate Distributions, Vol. 2, $2^{\text {nd }}$ ed., 1995, (Houghton Mifflin: Boston).

Kelle, P., A. Milne, The effect of (s, S) ordering policy on the supply chain, Int. J. Prod. Econ.,1999, 59(1), 113 - 122.

Kreipl, S., M. Pinedo, Planning and scheduling in supply chains: an overview of Issues in Practise, Prod. Op. Manag., 2004, 13(1), 77 - 92.

Li, J., M. Shaw, R. Sikora, G. W. Tan, R. Yang, The effects of Information sharing strategies on supply chain performance, Working paper, University of Illinois Urbana-Champaign, 2001.

Longo, F., G. Mirabelli, E. Papoff, Modelling Analysis and Simulation of a supply chain devoted to support pharmaceutical business retail, in $18^{\text {th }}$ International Conference on Production Research, 2005.

Longo F., G. Mirabelli, E. Papoff, Modeling, Analysis \& Simulation of Tubes Manufacturing Process and Industrial Operations Controls, in Summer Computer Simulation Conference, 2006 pp.54- 59 .

Parker, R.P., R. Kapuscinski, Optimal Policies for a Capacitated Two-Echelon Inventory system, Op. Res., 2004, 52(5), $739-755$.

Roundy, R. O., J. A. Muckstadt, Heuristic computation of periodic-review base stock inventory policies, Manag. Sc., 2000, 46(1), $104-109$.

Simchi-Levi, D., P. Kaminsky, E. Simchi-Levi, Designing and managing the supply chain, 2000, (McGraw-Hill: Illinois).

Stadtler, H., C. Kilger, Supply chain management and advanced planning, 2000 (Springer: Berlin).

Suwanruji P., S.T. Enns, Evaluating the effects of capacity constraints and demand patterns on supply chain replenishment strategies, Int. J. of Prod. Res., 2006, 44(21), pp. 4607 - 4629.

Towill, D. R., Supply chain dynamics, Int. J. Comp. Integr. Manuf., 1991, 4(4), 197 - 208.

Wikner, J., D. R. Towill, M. Naim, Smoothening supply chain dynamics, Int. J. Prod. Econ., 1991, 3(3), $3-13$.

Zipkin, P., Foundations of Inventory Management, 2000, (McGraw-Hill Higher Education: Boston). 
Table 1. Data Collection in each supply chain node

\begin{tabular}{|c|c|c|}
\hline Plants & Distribution centres & Retailers \\
\hline List of processes and activities & List of processes and activities & List of processes and activities \\
Process Time & Lead Time & Demand arrival process \\
Setup Time & Inventory Control Policy & Customer demand \\
Lead Time & Forecast Method & Lead Time \\
Number and type of machines & Inventory Costs & Inventory Control Policy \\
Bill of materials & Items mixture & Forecast Method \\
Items mixture & & Inventory Costs \\
& & Items mixture \\
\hline
\end{tabular}


Table 2: Goodness of fit tests results and distribution parameters for inter-arrival times (retailer \#1)

\begin{tabular}{|c|c|c|c|c|c|}
\hline Distributions & Chi Statistics & Chi Value & Results & Parameter 1 & Parameter 2 \\
\hline Erlang & 18.419 & 24.997 & true & 4163.164 & 4163.164 \\
\hline Weibull & 25.925 & 24.997 & false & 1.009 & 4184.344 \\
\hline Negexp & 16.315 & 26.297 & true & 4168.058 & \\
\hline Lognorm & 129.001 & 24.997 & false & 5383.540 & 11142.929 \\
\hline
\end{tabular}


Table 3. Statistic distributions and parameters

\begin{tabular}{|c|c|c|}
\hline Variables & Distribution Type & Parameters estimation \\
\hline Inter-arrival time & Neg. Expon. (Poisson) & $\mathrm{m}=1.16$ hours (mean inter arrival time) \\
\hline Quantity (item\#1) & Triangular & $\mathrm{min}=21$, mean $=30, \max =40$ pallets \\
\hline Item mixture & Empirical & $\mathrm{m}=2$ days (mean value); $=0.4$ days (stand. dev.) \\
\hline Lead Time (Plants) & Gaussian & $\mathrm{m}=3$ days (mean value) $\mathrm{s}=0.5$ days (stand. dev.) \\
\hline Lead Time (DCs) & Gaussian & \\
\hline Process Time (Plants) & Empirical & \\
\hline Setup Time (Plants) & Empirical & \\
\hline
\end{tabular}


Table 4: Experimental Design: factors and levels

\begin{tabular}{|l|cccc|}
\hline & $\begin{array}{c}\text { F1 - Inventory Control } \\
\text { Policy }\end{array}$ & F2 - Lead Time & $\begin{array}{c}\text { F3 - Demand } \\
\text { Intensity }\end{array}$ & $\begin{array}{c}\text { F4 - Demand } \\
\text { Variability }\end{array}$ \\
\hline Level 1 - L1 & ICP1 - actual at retailers & 1 day - new scenario & Low - new scenario & Low - new scenario \\
Level 2 - L2 & ICP2 - new scenario & 3 days - actual & Medium - actual & Medium - actual \\
Level 3 - L3 & ICP3 - actual at DCs & 5 days - new scenario & High - new scenario & High - new scenario \\
Level 4 - L4 & ICP4 - new scenario & & & \\
\hline
\end{tabular}


Table 5: $1^{\text {st }}$ scenario analysis (retailer \#1)

\begin{tabular}{|c|cccc|}
\hline Scenarios & F1 - L1 & F1 - L2 & F1 - L3 & F1 - L4 \\
& (ICP1) & (ICP2) & (ICP4) & (ICP4) \\
\hline Low Intensity & 0.660 & 0.509 & 0.707 & 0.749 \\
(pessimistic) & $(74)$ & $(84)$ & $(40)$ & $(30)$ \\
Medium Intensity & 0.396 & 0.167 & 0.428 & 0.429 \\
(actual) & $(78)$ & $(90)$ & $(51)$ & $(45)$ \\
High Intensity & 0.224 & 0.046 & 0.234 & 0.237 \\
(optimistic) & $(114)$ & $(144)$ & $(99)$ & $(94)$ \\
\hline
\end{tabular}


Table 6: $2^{\text {nd }}$ scenario analysis (retailer \#1)

\begin{tabular}{|c|cccc|}
\hline Scenarios & F1 - L1 & F1 - L2 & F1 - L3 & F1 - L4 \\
& (ICP1) & (ICP2) & (ICP3) & (ICP4) \\
\hline Low Variability (optimistic) & 0.406 & 0.174 & 0.452 & 0.457 \\
& $(75)$ & $(83)$ & $(50)$ & $(43)$ \\
Medium Variability (actual) & 0.394 & 0.163 & 0.423 & 0.429 \\
& $(77)$ & $(84)$ & $(51)$ & $(44)$ \\
High Variability (pessimistic) & 0.387 & 0.151 & 0.413 & 0.403 \\
& $(82)$ & $(87)$ & $(54)$ & $(46)$ \\
\hline
\end{tabular}


Table 7: $3^{\text {rd }}$ scenario analysis (retailer \#1)

\begin{tabular}{|c|cccc|}
\hline Scenarios & F1 - L1 & F1 - L2 & F1 - L3 & F1 - L4 \\
\hline 1 day Lead Time & 0.405 & 0.196 & 0.432 & 0.436 \\
(Optimistic) & $(62)$ & $(68)$ & $(37)$ & $(35)$ \\
3 days Lead Time & 0.394 & 0.163 & 0.423 & 0.429 \\
(Actual) & $(77)$ & $(84)$ & $(51)$ & $(44)$ \\
5 days Lead Time & 0.355 & 0.121 & 0.418 & 0.419 \\
(pessimistic) & $(90)$ & $(90)$ & $(58)$ & $(50)$ \\
\hline
\end{tabular}


Figure 1: Supply chain schematization

Figure 2: Inter-arrivals times of retailer \#1: real data and distribution fitting Figure 3: Main frame of the simulation model

Figure 4: Mean Square pure Error analysis (distribution centre \#2)

Figure 5: Comparison between real and simulated fill rate curves for retailer \#1

Figure 6: $1^{\text {st }}$ scenario, inventory control policies comparison (retailer \#1)

Figure 7: $2^{\text {nd }}$ Scenario, inventory control policies comparison (retailer \#1)

Figure 8: $3^{\text {rd }}$ Scenario, inventory control policies comparison (retailer \#1)

Manuscript ID: TPRS-2007-IJPR-0071.R1 


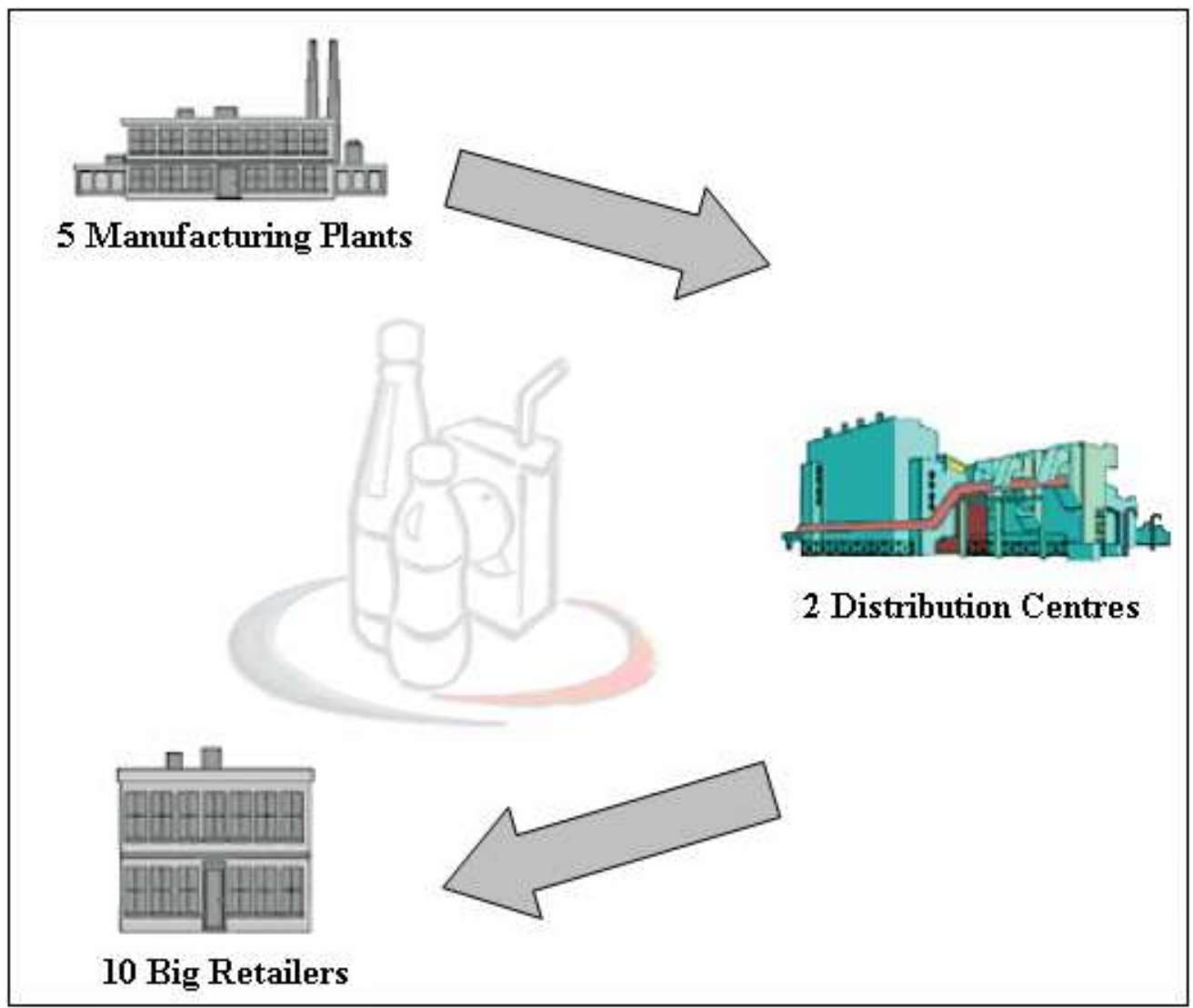

Supply chain schematization

$137 \times 116 \mathrm{~mm}$ (96 x 96 DPI) 


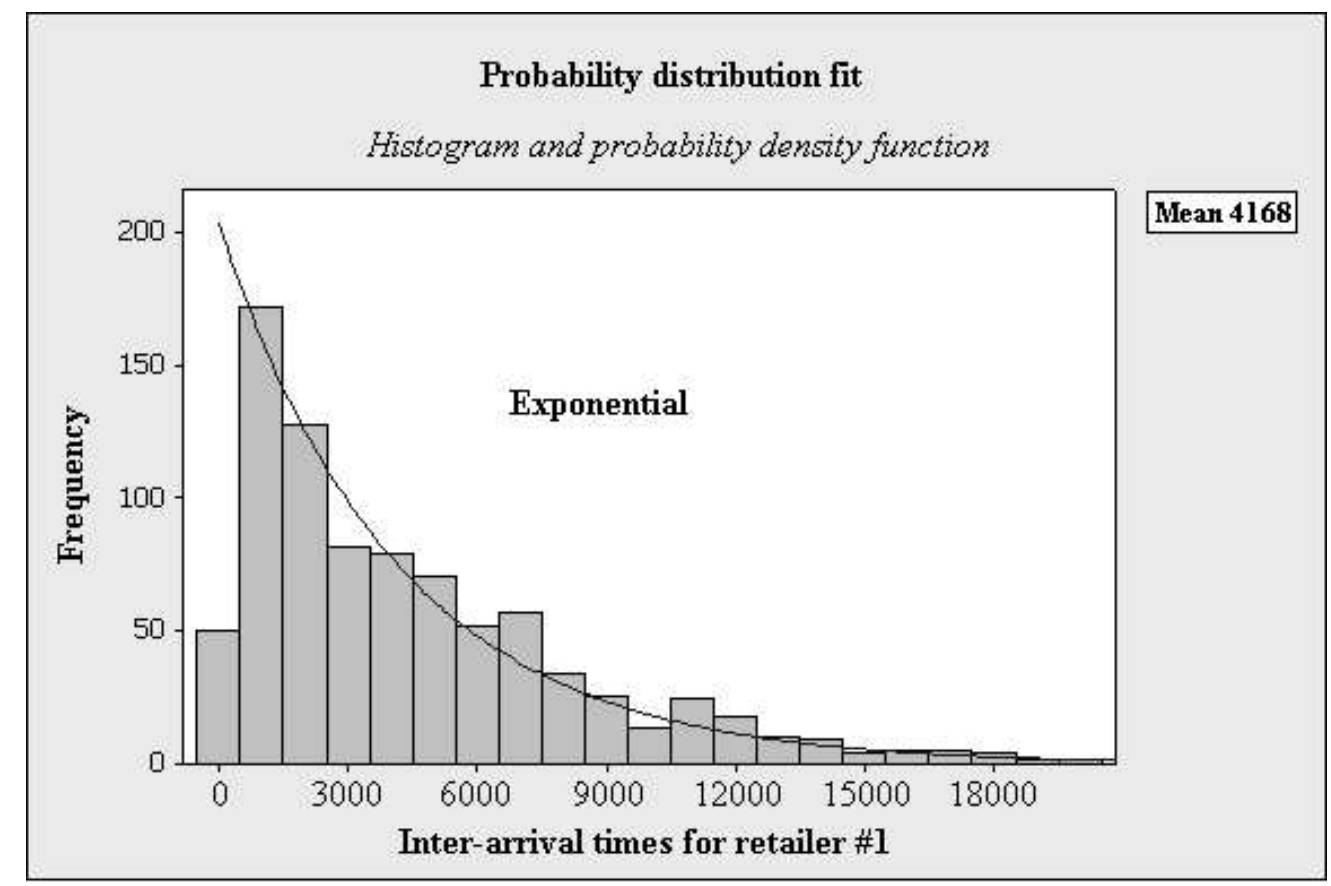

Inter-arrivals times of retailer \#1: real data and distribution fitting $203 \times 135 \mathrm{~mm}(72 \times 72 \mathrm{DPI})$ 


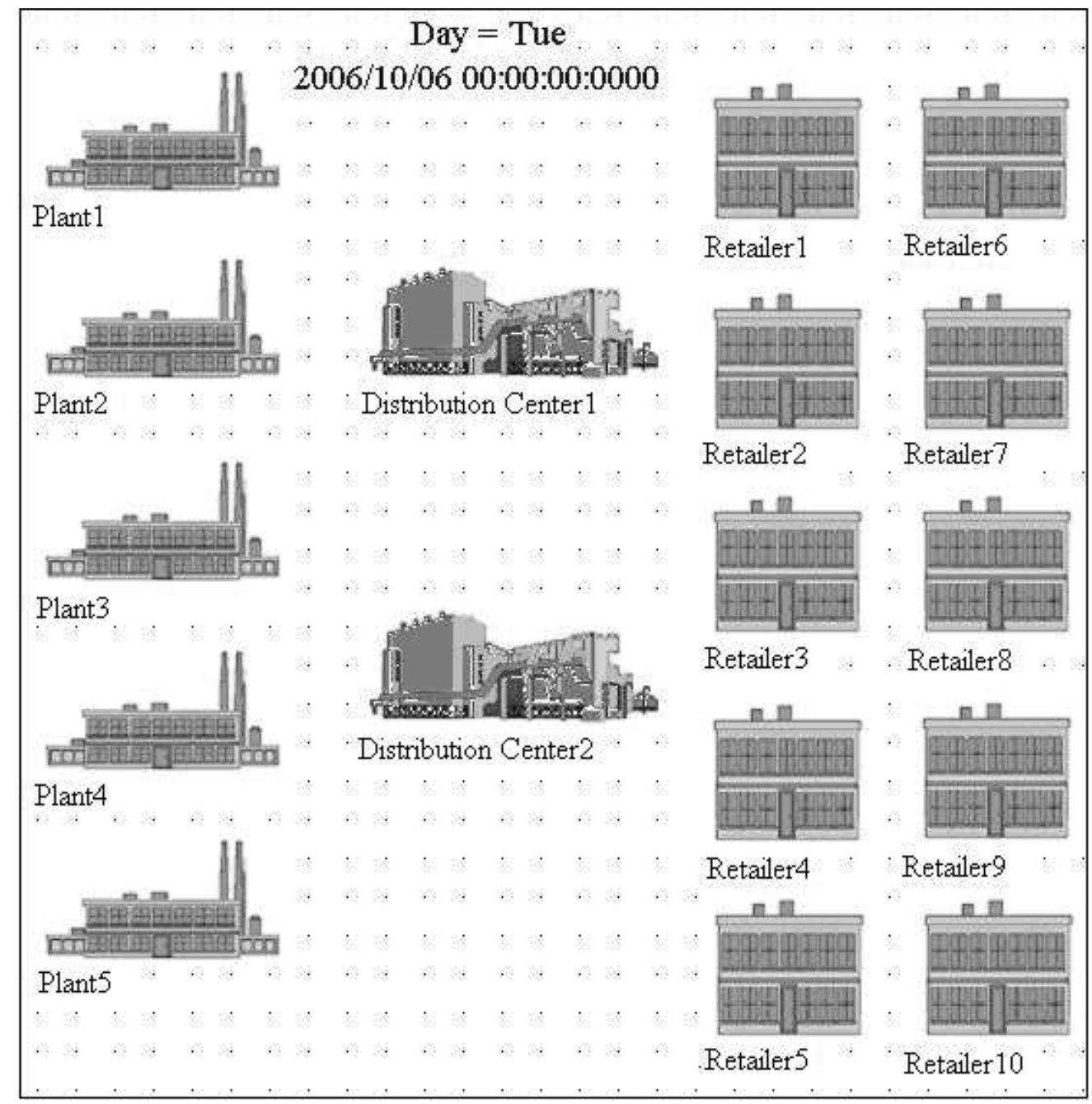

Main frame of the simulation model $195 \times 199 \mathrm{~mm}(72 \times 72 \mathrm{DPI})$ 


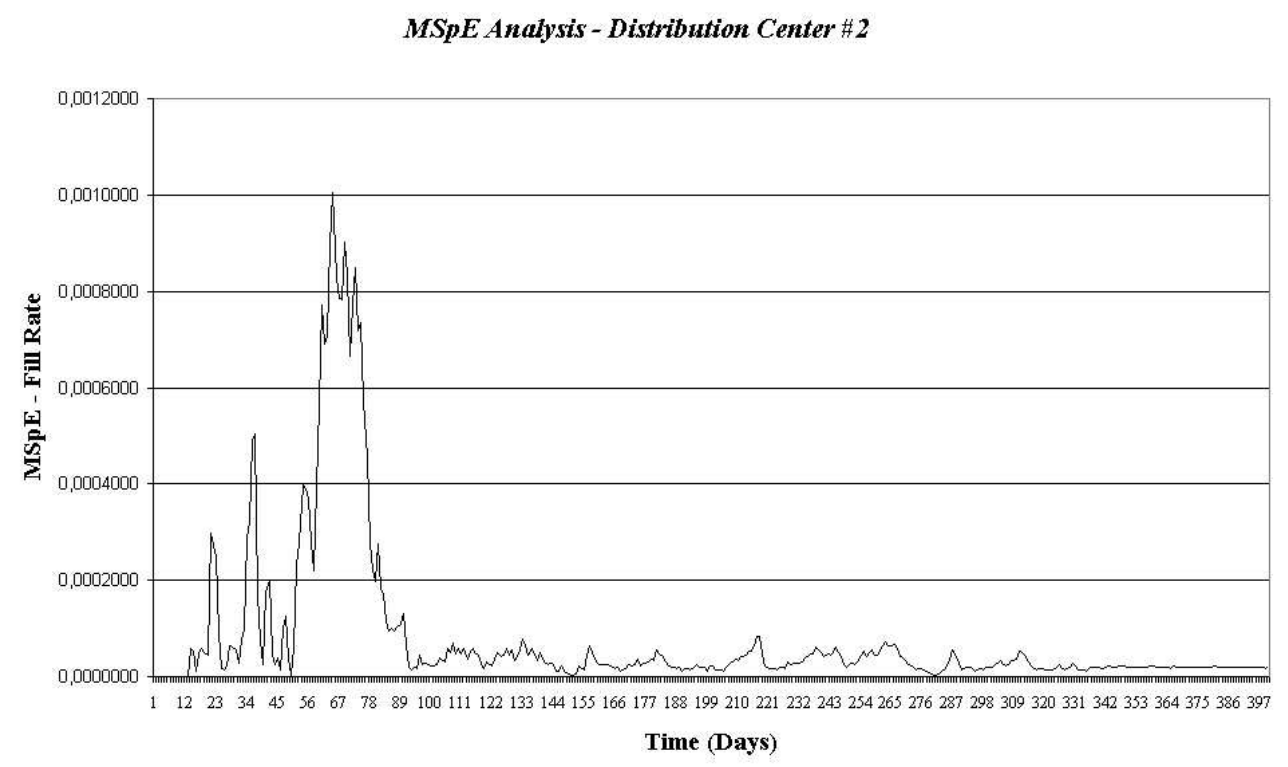

Mean Square pure Error analysis (distribution centre \#2) $342 \times 212 \mathrm{~mm}(72 \times 72 \mathrm{DPI})$ 


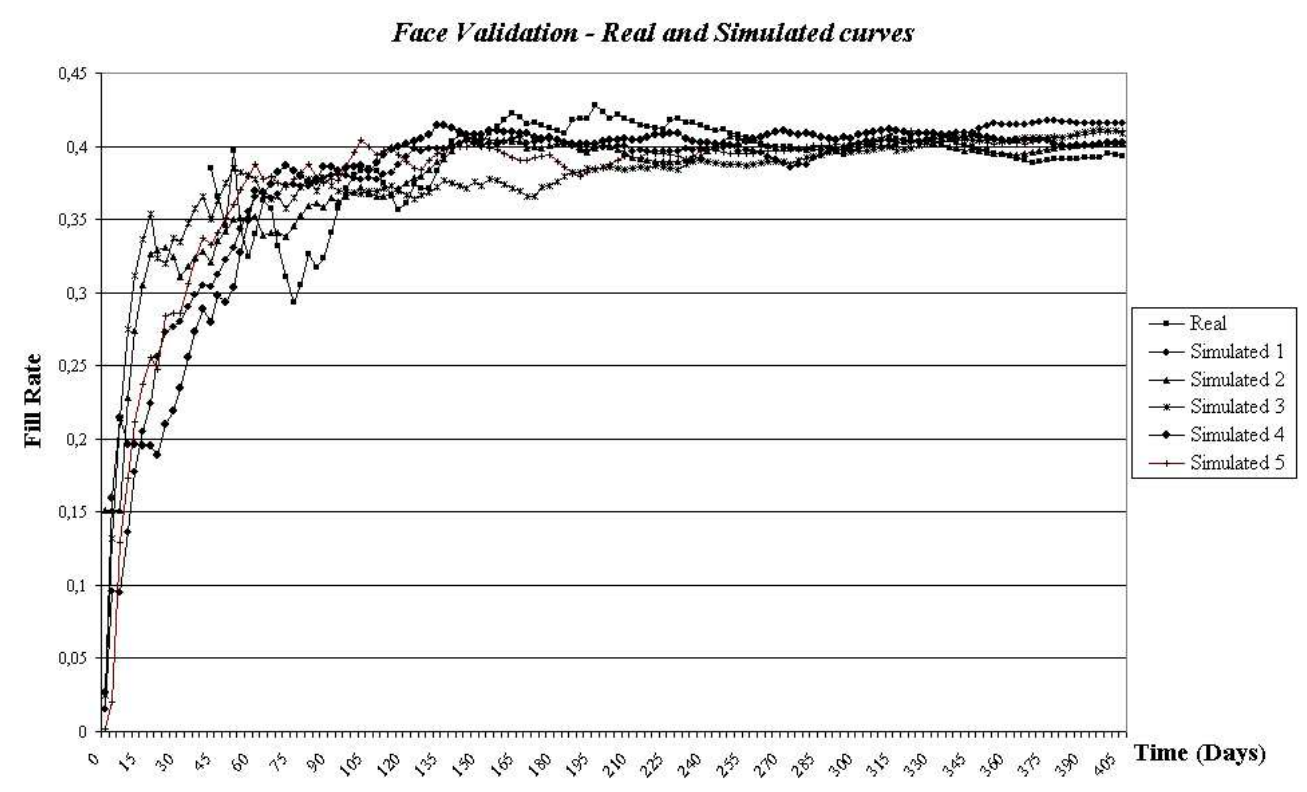

Comparison between real and simulated fill rate curves for retailer \# 1 $342 \times 212 \mathrm{~mm}(72 \times 72 \mathrm{DPI})$

http://mc.manuscriptcentral.com/tprs Email: ijpr@lboro.ac.uk 


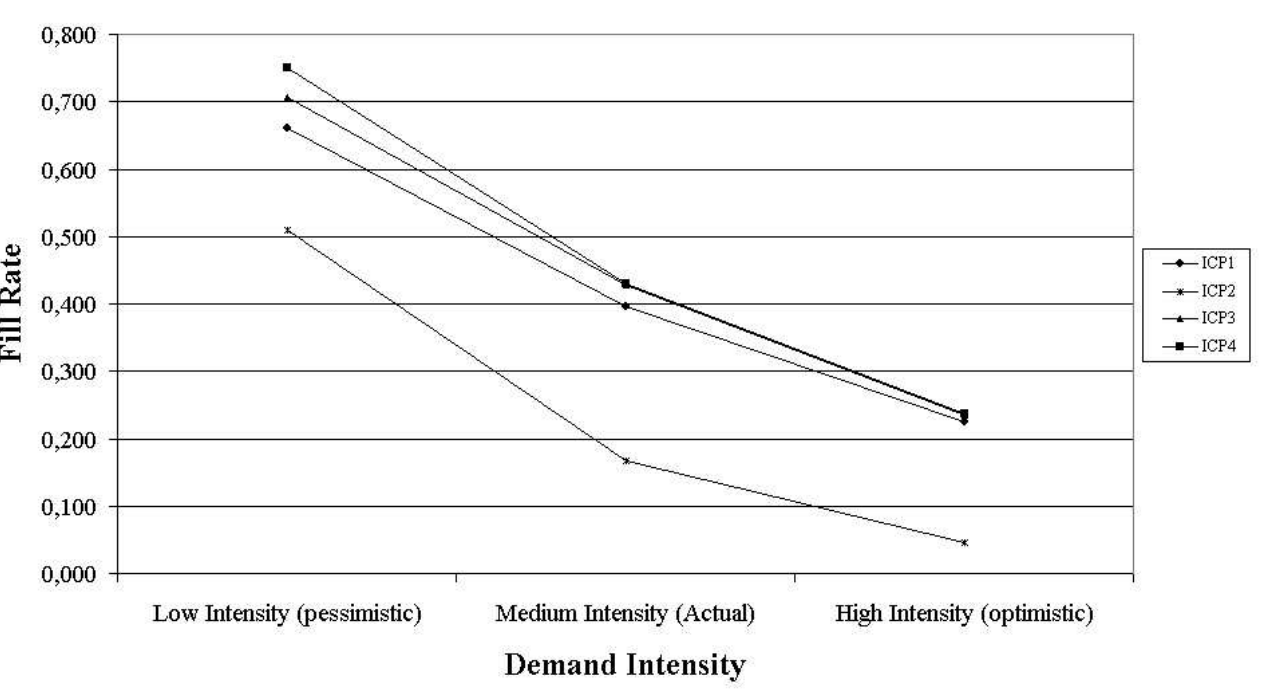

1st scenario, inventory control policies comparison (retailer \#1) $342 \times 212 \mathrm{~mm}(72 \times 72 \mathrm{DPI})$ 
Scenario 2

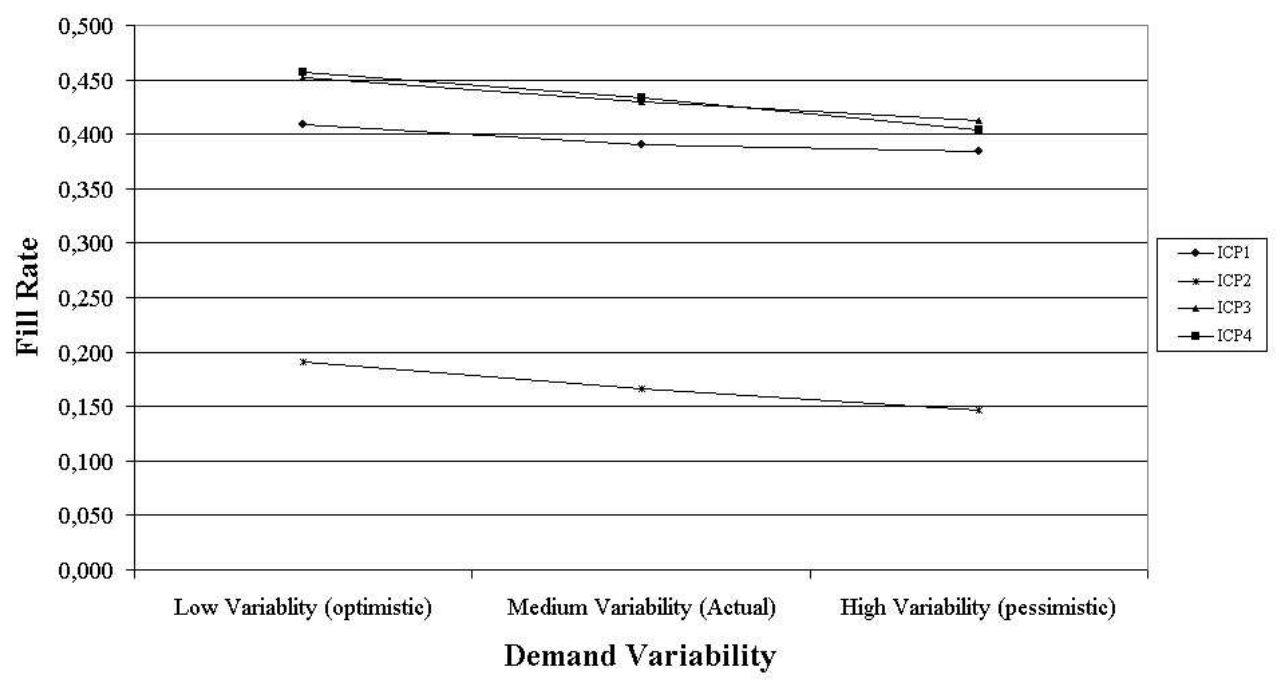

2nd Scenario, inventory control policies comparison (retailer \#1) $342 \times 212 \mathrm{~mm}(72 \times 72$ DPI $)$

http://mc.manuscriptcentral.com/tprs Email: ijpr@lboro.ac.uk 


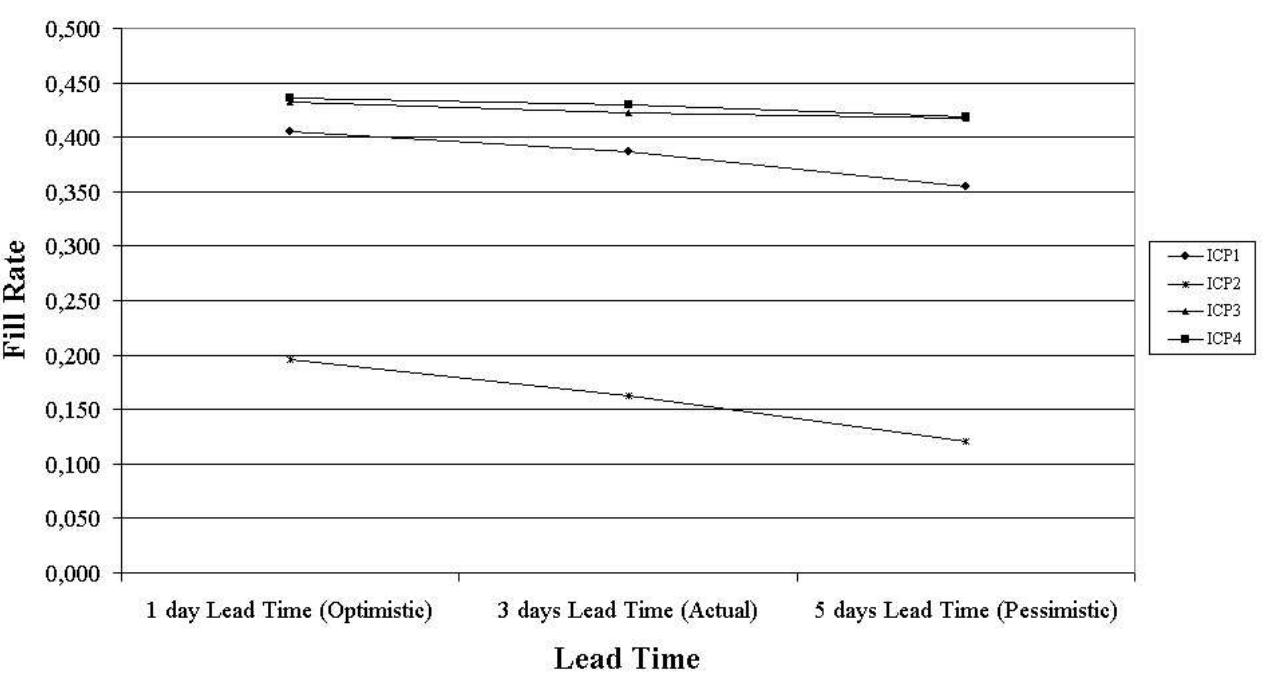

3rd Scenario, inventory control policies comparison (retailer \#1) $342 \times 212 \mathrm{~mm}(72 \times 72$ DPI $)$ 